\title{
Disciplinary Challenges of Today Science
}

\author{
Octavio A Chon-Torres* \\ Professor at Universidad de Lima, Doctorate professor at Universidad Nacional Mayor de San Marcos, peru
}

Received: April 14, 2018; Published: April 19, 2018

*Corresponding author: Octavio A Chon-Torres, President of Asociación Peruana de Astrobiología, Member of the International Working Group on Astrobioethics, Organizer of the IV International Astrobiology Congress, peru, Tel: 51 995658337; Email: ochon@ulima.edu.pe;octavio@octaviochon.com

\section{Opinion}

We are currently witnessing a series of events that, more than isolated or separate problems are actually interconnected, complex, with a content of variables that exceed the object of study of a single scientific or academic discipline. We talk about, for example, the environmental problem, the public health, and even astrobiology (study of life in the universe). What do these and other more scenarios have in common that can draw attention to a new approach of dealing with complex problems? First of all, we must bear in mind that science is a tool; as such it requires philosophy to be able to understand itself. Hence, words such as paradigm or falsification of science, although it is true they help us to understand the process and evolution of scientific knowledge, are not strictly definitions that come out of the scientific method, but rather it is philosophical terminology.

Well, science needs a context to develop. This is strongly influenced by the culture of the time, so we can understand that the concept of science 500 years ago is different from what we have today. The study of science objects respond to those that society needs to solve for its survival. 500 years ago, the scientific community was not particularly interested in inhabiting Mars. Now it is a subject of study and a possibility on which we depend as humanity.The ability for a discipline or researcher to adapt to other disciplinary approaches is crucial for the successful development of their research. We talk about multi, inter and transdisciplinary approaches. But what are we talking about when we refer to these approaches? Let's take the first one; a multidisciplinary approach is one that manages to bring together different specialists in a team. Perhaps among them they never achieve a close interaction, but the highlight in this way of investigating is that each discipline maintains its own methodology. Although it is true that science has the scientific method, it acquires different nuances according to the nature of the object of study.

For example, the behavior of a person will not be equally methodologically studied when analyzing the chemical composition of water.Even the terminology is different, the principles and rules that govern each discipline or nomological network determine the interpretation and result of it. So, multidisciplinary extends the horizon but does not interconnect the terrain. It makes us see variables and problems where other disciplines fail to arrive, but it does not establish the interconnected roadmap of action and methodological exchange that is required to be able to deal appropriately with this kind of problems. However, we see that the word multidisciplinary can be very used when it comes to relating disciplines. We now know that this belongs to an initial level of disciplinary relationships.

On the other hand, interdisciplinarity is the joint activity of several disciplines, but the central point of study is directed by a discipline. That is, several fields of study come together and contribute their work methodology to increase knowledge of the science that directs the research. This is the case of the philosophy of biology, for example, which, without being biology, uses conceptual inputs from biology to be able to work on philosophical problematization. The same could be said of psychiatry, which considers the behavior of people in order to determine the need to supply drugs that have an effect on the human brain and then on its behavior. These are ways to increase the quality of knowledge, complementing it with other approaches, but which nevertheless remains an approach that is limited to the orientation of one discipline. Multidisciplinary and interdisciplinarity, therefore, are two ways of seeing the world that have something in common, and that is the specialization of knowledge centered parts.

Let's consider the following case: the environmental problem. There ir not enough emphasis on biological aspects to understand this situation. A multidisciplinary approach can help us understand the different edges that this problem can present, but its level of complexity will not allow us to take adequate measures from the multidisciplinary approach. If we approach through interdisciplinarity, this will give us as a result the increase of knowledge of a certain area in particular, but it will not be enough either. The case of environmental problems is too complex to be addressed by these approaches, and the same could be said of public health, so in this last case, in addition to biological factors, the 
psychological, economic, environmental, religious, etc. The union and contribution of each can help us understand what happens, but we need to connect them in a way that methodologically speaking has successful results.

The so-called transdisciplinarity is presented as the most realistic and appropriate option to address this type of polyproblems. The prefix "trans" means "through", so that the transdisciplinary solution is the one that passes through all the disciplines involved. The transdisciplinary methodology should not be limited to one specialty, what it needs is to connect the problems of all the disciplines involved and draw up an action map, a road map for the situation that is presented to us.It is the same thing that happens, in another case, with astrobiology, because we don't have a theory of the origin of life in the universe, works according to the developing progress. In a transdisciplinary approach, which would be what Gibbons et al. In the book the new production of knowledge in 1994 we are known as Mode 2 of knowledge production, teamwork is essential, but more essential is the team mentality, because we can bring together different specialists to work on a topic.

The challenge here is to connect the mentalities of the specialists to produce an orchestrated work method. Therefore, we can consider this mode of knowledge as a posteriori, and more than a pre-established theory, it is a way of working that has not yet defined its dynamism. It is essential, therefore, that the criteria of transdisciplinarity be established. This is a work that starts with the philosophical considerations of the same and evolves in concrete forms of work. The observation and attention to the new challenges facing the disciplinary nature of science may even end up becoming a new, if one want to call it, paradigm. Therefore, the disciplinary challenges of current science are those that society faces, and to be able to do it successfully it is important to change our mentality and way of approaching the objects of our study.

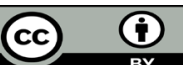

This work is licensed under Creative Commons Attribution 4.0 License

Submission Link: https://biomedres.us/submit-manuscript.php

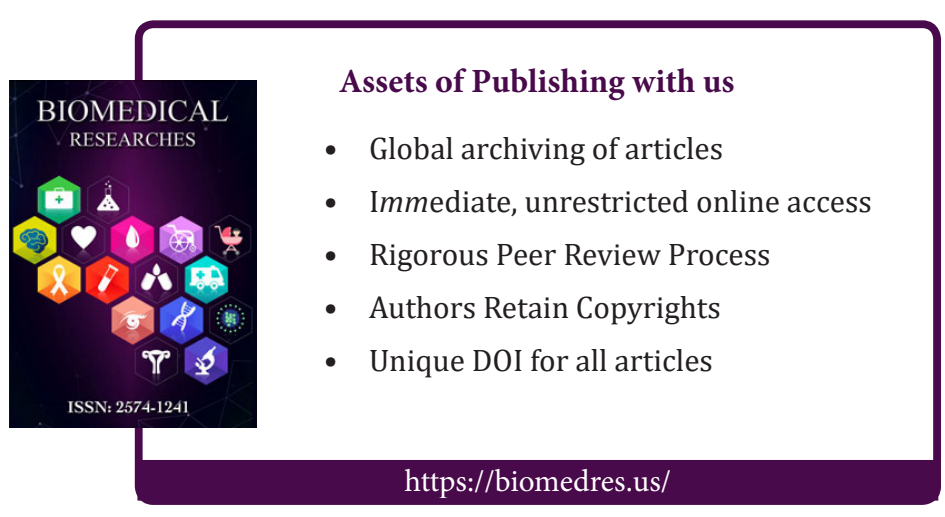

